\begin{tabular}{r|r|}
$H$ & $\substack{\text { 1. ECN } \\
\text { Page 1 of } 2}$ \\
\hdashline$\substack{\text { Proj. } \\
\text { ECN }}$ & 653347 \\
\hline
\end{tabular}

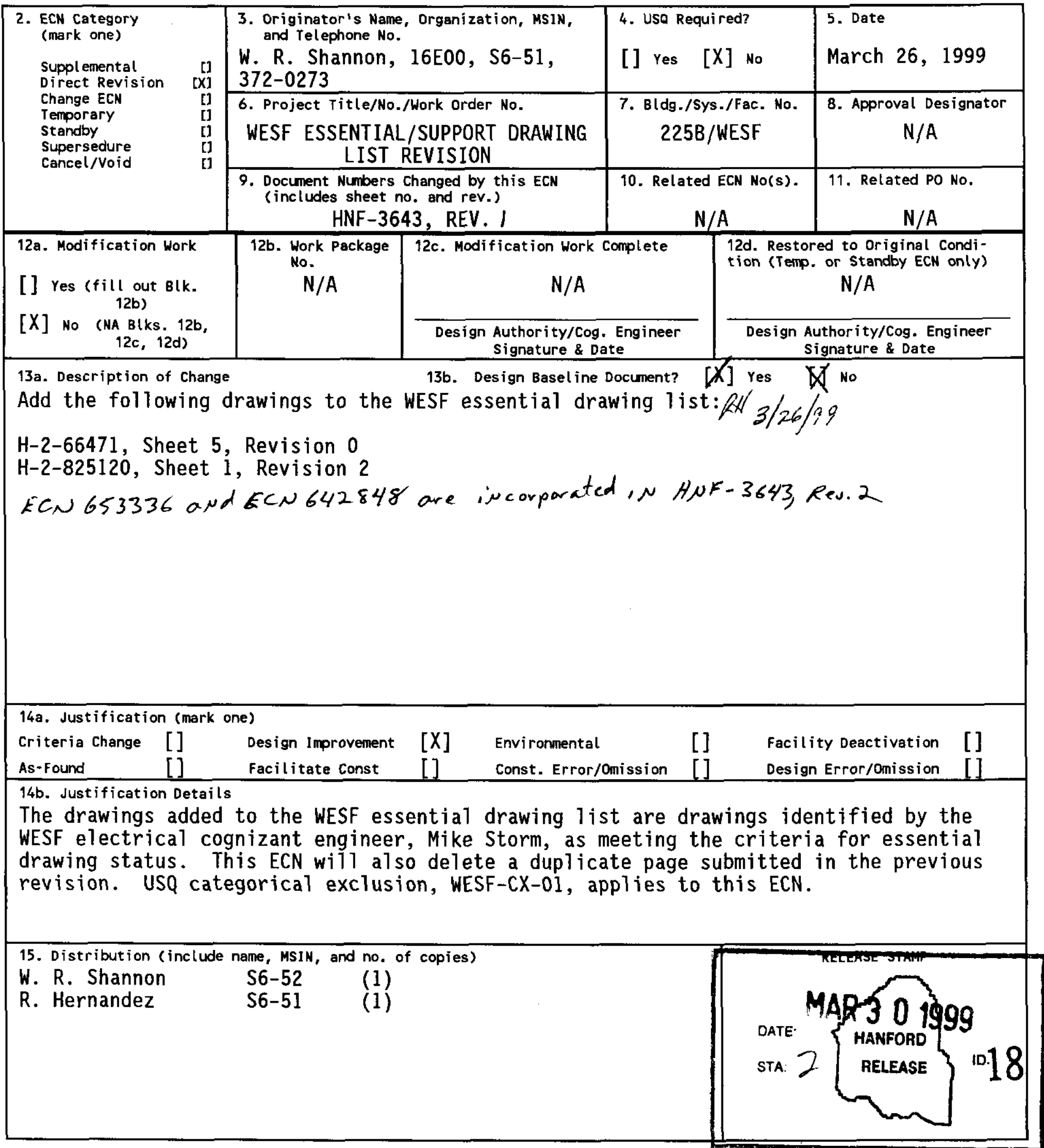




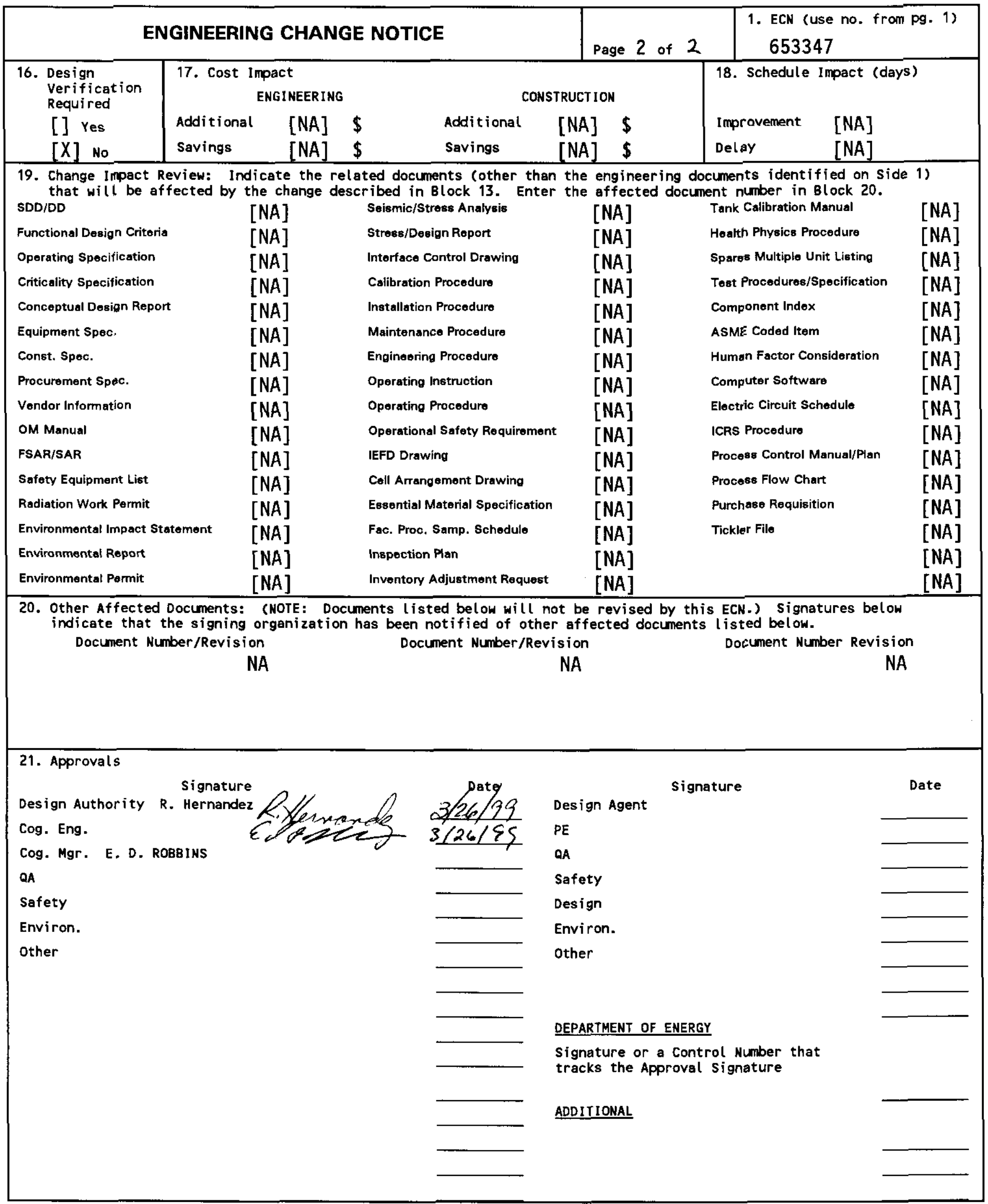




\section{WASTE AND ENCAPSULATION STORAGE FACILITY ESSENTIAL AND SUPPORT DRAWING LIST}

Wayne R. Shannon

B \& W Hanford Co, Richland, WA 99352

U.S. Department of Energy Contract DE-AC06-96RL13200

EDT/ECN: 650674

Org Code: 16E00

B\&R Code: EW7002010

UC: 508

Charge Code: 101279

Total Pages: 8

Key Words: Essential, Support, WESF, Essential drawing list, Support drawing list

Abstract: Provides listing of Essential and Support Drawings for the Waste and Encapsulation Storage Facility.

TRADEMARK DISCLAIMER. Reference herein to any specific commercial product, process, or service by trade name, trademark, manufacturer, or otherwise, does not necessarily constitute or imply its endorsement, recommendation, or favoring by the United States Government or any agency thereof or its contractors or subcontractors.

Printed in the United States of America. To obtain copies of this document, contact: Document Control Services, P.O. Box 950, Mailstop H6-08, Richland WA 99352, Phone (509) 372-2420; Fax (509) 376-4989.
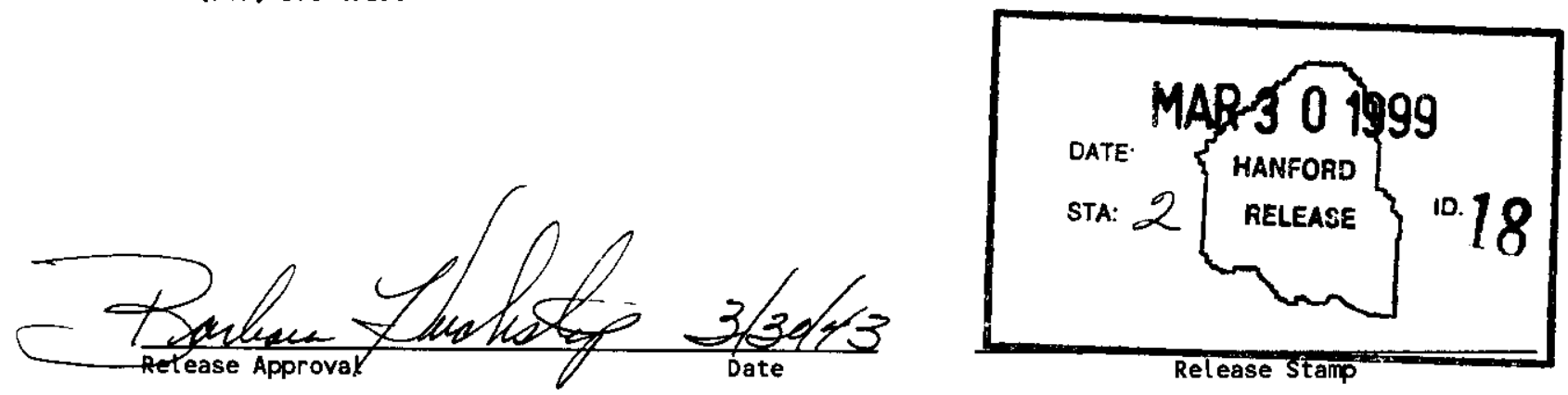

\section{Approved for Public Release}


(2) Title

Waste and Encapsulation Storage Facility essential and support drawing list CHANGE CONTROL RECORD

\begin{tabular}{|c|c|c|c|c|}
\hline \multirow{2}{*}{ (3) } & \multirow{2}{*}{ Revision } & \multirow{2}{*}{ (4) Description of Change - Replace, Add, and Delete Pages } & \multicolumn{2}{|c|}{ Authorized for Release } \\
\hline & & & (5) Cog. Engr. & (6) Cog. Mgr. \\
\hline & 0 & (7) Released by EDT 625880 on $11 / 24 / 98$ & W R Shannon & $\begin{array}{l}\text { E D Robbins } \\
11 / 24 / 98\end{array}$ \\
\hline & 1 & Revised by ECN 650674 & W R Shannon & E D Robbins \\
\hline & & Revised by ECN $653347, E C N 653336$, ECN 642848 & 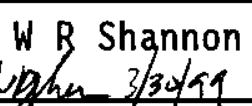 & ED Robbins \\
\hline
\end{tabular}




\section{Waste and Encapsulation Storage Facility (WESF) essential and support drawing list}

The drawings identified in this document will comprise the Waste Encapsulation and Storage Facility essential and support drawing list. This list will replace drawings identified as the "WESF Essential and support drawing list". Additionally, this document will follow the applicable requirements of HNF-PRO-242 " Engineering Drawing Requirements" and FSP-WESF-001, Section EN-1 "Documenting Engineering Changes".

An essential drawing is defined as an engineering drawing identified by the facility staff as necessary to directly support the safe operation or maintenance of the facility. A support drawing is defined as a drawing identified by the facility staff that further describes the design details of structures, systems, or components shown on essential drawings or is frequently used by the support staff.

As a minimum, essential drawings should be considered from the following types:

\section{Process Systems}

- Process and Instrument Diagrams (P\&ID)

- Instrument and Engineering Flow Diagrams (IEFD)

- Required Elementary/Schematic Drawings

- One-Line Diagrams

- Process Flow Sheets

\section{Electrical System}

- One-Line Diagrams

- Panel Schedules

- Power and Lighting Diagrams

\section{Effluent Monitoring Systems}

- Process and Instrument Diagrams

\section{HVAC System}

- One-Line Diagrams

- Elementary/Schematic Diagrams

- Process and Instrument Diagrams (P\&ID)

\section{Civil/Structural}

- Confinement/Containment

- Anchorage 


\section{Steam System}

- Steam Distribution Drawings

- Process and Instrument Diagrams (P\&ID)

\section{Fire Protection System}

- Detector Scheme Drawings

- Elementary/Schematic Diagrams

- RFAR Control Scheme Diagrams

\section{$\underline{\text { Air/Water Distribution System }}$}

- High Pressure Process and Instrument Diagrams (P\&ID)

\section{Communication/Alarm Systems (Criticality, Evacuation, etc)}

- Elementary Diagrams

- One-Line Diagrams

- Detector Scheme Diagrams

Any of the following conditions can result in a drawing being defined as essential. The drawing is:

1. Essential to the safe operation and understanding of facility process and support systems. Loss of drawing integrity results in an increased potential for process misroutings and false process parameter indication.

2. Accurate ready-reference for facility operations personnel required for response during upset conditions (especially those called out by emergency or alarm response procedures).

3. Drawings used to demonstrate compliance with Federal, State, and DOE required laws, rules, and regulations.

4. Loss of drawing integrity results in increased potential for failure of emergency notification and alarm systems.

5. Drawings used (especially in the case of electrical or high pressure fluid systems) to determine proper boundary isolation for personnel protection while working on deenergized systems.

6. Drawings that depict and identify instrumentation required by authorization basis documentation. 
Drawings should be removed from essential/support status if any of the following occur:

7. Drawing is voided, or superseded by another drawing.

8. Drawing represents a system that no longer exists, has been inactivated, or its function no longer meets any of the criteria stated above. 


\section{WESF ESSENTIAL DRAWINGS}

\begin{tabular}{|c|c|c|c|c|}
\hline & Document Number & Sheet & $\operatorname{Rev}$ & Title \\
\hline 1 & $\mathrm{H}-2-66400$ & 3 & 10 & DRAWING INDEX \\
\hline 2 & & 4 & 4 & DRAWING INDEX \\
\hline 3 & & 5 & 4 & DRAWING INDEX \\
\hline 4 & $\mathrm{H}-2-66402$ & 1 & 4 & ARCHITECTURAL FIRST FLOOR PLAN AREA 2 \\
\hline 5 & $\mathrm{H}-2-66403$ & 1 & 5 & ARCHITECTURAL FIRST FLOOR PLAN AREA 3 \\
\hline 6 & $\mathrm{H}-2-66471$ & 1 & 14 & ELECTRICAL LIGHTING PANEL SCHEDULE \\
\hline 7 & & 2 & 12 & ELECTRICAL LIGHTING PANEL SCHEDULE \\
\hline 8 & & 3 & 12 & ELECTRICAL LIGHTING PANEL SCHEDULE \\
\hline 9 & & 4 & 2 & ELECTRICAL LIGHTING PANEL SCHEDULE \\
\hline 10 & & 5 & 0 & ELECTRICAL LIGHTING PANEL SCHEDULE \\
\hline 11 & $\mathrm{H}-2-66482$ & 1 & 8 & ELECTRICAL FIRE ALARM DIAGRAM \\
\hline 12 & & 2 & 0 & ELECTRICAL FIRE ALARM DIAGRAM \\
\hline 13 & & 3 & 2 & ELECTRICAL FIRE ALARM DIAGRAM \\
\hline 14 & $\mathrm{H}-2-66531$ & 1 & 9 & HVAC AIR FLOW DIAGRAM \\
\hline 15 & $\mathrm{H}-2-66532$ & 1 & 13 & HVAC CONTROL DIAGRAM \\
\hline 16 & & 2 & 4 & HVAC CONTROL DIAGRAM \\
\hline 17 & & 3 & 4 & HVAC CONTROL DIAGRAM \\
\hline 18 & $\mathrm{H}-2-66533$ & 1 & 10 & HVAC CONTROL DIAGRAM \\
\hline 19 & & 2 & 15 & HVAC CONTROL DIAGRAM \\
\hline 20 & $\mathrm{H}-\overline{2-66681}$ & 1 & 10 & INSTRUMENT ENGINEERING DIAGRAM BUILDING EXHAUST SYSTEM K1 \\
\hline 21 & $\mathrm{H}-2-66682$ & 1 & 9 & INSTRUMENT ENGINEERING DIAGRAM CELL EXHAUST SYSTEM K3 \\
\hline 22 & $\mathrm{H}-2-66986$ & 1 & 11 & ENGINEERING FLOW DIAGRAM POOL CELL 1 \\
\hline 23 & & 10 & 3 & ENGINEERING FLOW DIAGRAM POOL CELL 10 \\
\hline 24 & & 11 & 6 & ENGINEERING FLOW DIAGRAM POOL CELL 11 \\
\hline 25 & & 12 & 2 & ENGINEERING FLOW DIAGRAM POOL CELL 12 AND DETAILS \\
\hline 26 & & 13 & 2 & ENGINEERING FLOW DIAGRAM DETAILS \\
\hline 27 & & 14 & 0 & ENGINEERING FLOW DIAGRAM DETAIL F \\
\hline 28 & & 15 & 10 & ENGINEERING FLOW DIAGRAM DETAIL G \\
\hline 29 & & 16 & 0 & ENGINEERING FLOW DIAGRAM DETAIL H \\
\hline 30 & & 17 & 0 & ENGINEERING FLOW DIAGRAM DETAIL J \\
\hline 31 & & 18 & 0 & ENGINEERING FLOW DIAGRAM DETAIL K \\
\hline 32 & & 2 & 8 & ENGINEERING FLOW DIAGRAM POOL CELL 2 \\
\hline 33 & & 3 & 5 & ENGINEERING FLOW DIAGRAM POOL CELL 3 \\
\hline 34 & & 4 & 6 & ENGINEERING FLOW DIAGRAM POOL CELL 4 \\
\hline 35 & & 5 & 4 & ENGINEERING FLOW DIAGRAM POOL CELL 5 \\
\hline 36 & & 6 & 3 & ENGINEERING FLOW DIAGRAM POOL CELL 6 \\
\hline 37 & & 7 & 3 & ENGINEERING FLOW DIAGRAM POOL CELL 7 \\
\hline 38 & & 8 & 3 & ENGINEERING FLOW DIAGRAM POOL CELL 8 \\
\hline 39 & & 9 & 3 & ENGINEERING FLOW DIAGRAM POOL CELL 9 \\
\hline 40 & $\mathrm{H}-2-66988$ & 1 & 8 & ENGINEERING FLOW DIAGRAM AQUEOUS MAKE-UP \\
\hline 41 & H-2-76583 & 1 & 3 & ARCH/FIRE PROT/PIPING FL PLANS EL 702'0" \\
\hline 42 & $\mathrm{H}-2-77735$ & 1 & 4 & COMPOSITE FLOW DIAGRAM AND SYSTEM DESCRIPTION \\
\hline 43 & & 2 & 4 & COMPOSITE FLOW DIAGRAM \\
\hline 44 & & 3 & 2 & COMPOSITE FLOW DIAGRAM BCE NEUTRALIZATION SYSTEM \\
\hline 45 & $\mathrm{H}-2-825120$ & 1 & 2 & MOTOR CONTROL CENTER 225BG-MCC-1 ONE LINE DIAGRAM \\
\hline 46 & $\mathrm{H}-2-825969$ & 1 & 5 & $\begin{array}{l}\text { FIRE PROTECTION SANITARY \& RAWWATEROISTRIBUTION KEY SITE } \\
\text { PIAN }\end{array}$ \\
\hline 47 & $\mathrm{H}-2-825972$ & 1 & 1 & FIRE PROT CONTROLS \& BARRIERS IST FLOOR PLAN \\
\hline 48 & & 2 & o & FIRE PROT CONTROLS \& BARRIERS 2ND \& MEZZ FLOOR PLAN \\
\hline
\end{tabular}




\begin{tabular}{|c|c|c|c|c|}
\hline 49 & & 3 & 2 & FIRE PROT CONTROLS \& BARRIERS SCHEDULES \\
\hline 50 & $\mathrm{H} \cdot 2-828987$ & 1 & 1 & P \& ID WESF LLLW SYSTEM SYMBOLS AND LEGENDS \\
\hline 51 & & 4 & 2 & P \& ID WESF LLLW SYSTEM \\
\hline 52 & & 5 & 1 & P \& ID WESF LLLW SYSTEM \\
\hline 53 & $\mathrm{H}-2-829004$ & 3 & 1 & ELECTRICAL SITE PLAN MCC 3 ONE-LINE \& ELEM \\
\hline 54 & $\mathrm{H}-2-829006$ & 2 & 1 & ELECTRICAL 225-BD \& TK-100 DET \& ELEV \\
\hline 55 & $\mathrm{H}-2-82967$ & 1 & 1 & CHEMICAL SEWER FLOW DIAGRAM \\
\hline 56 & $\mathrm{H}-2-92878$ & 1 & 3 & WINDOW PANEL ARRANGEMENT \\
\hline 57 & & 2 & 2 & WINDOW PANEL - ARRANGEMENT \\
\hline 58 & $\mathrm{H}-2-93127$ & 1 & 3 & ELECTRICAL-POOL CELL CONTAMINATION CONTROL SYSTEM \\
\hline 59 & & 2 & 1 & ELECTRICAL-POOL CELL CONTAMINATION CONTROL SYSTEM \\
\hline 60 & & 3 & 1 & ELECTRICAL-POOL CELL CONTAMINATION SYSTEM \\
\hline 61 & & 4 & 2 & ELECTRICAL-POOL CELL CONTAMINATION CONTROL SYSTEM \\
\hline 62 & & 5 & 1 & ELECTRICAL-POOL CELL CONTAMINATION CONTROL SYSTEM \\
\hline 63 & $\mathrm{H}-2-94500$ & 1 & 5 & INSTR ENGRG FLOW DIAGRAM CELL-A \\
\hline 64 & & 2 & 7 & INSTR ENGRG FLOW DIAGRAM CELL-A \\
\hline 65 & $\mathrm{H}-2-94501$ & 1 & 3 & INSTR ENGRG FLOW DIAGRAM CELL-B \\
\hline 66 & & 2 & 3 & INSTR ENGRG FLOW DIAGRAM CELL-B \\
\hline 67 & $\mathrm{H}-2-94502$ & 1 & 4 & INSTR ENGRG FLOW DIAGRAM CELL-C \\
\hline 68 & & 2 & 4 & INSTR ENGRG FLOW DIAGRAM CELL-C \\
\hline 69 & $\mathrm{H}-2-94503$ & 1 & 4 & INSTR ENGRG FLOW DIAGRAM CELL-D \\
\hline 70 & & 2 & 4 & INSTR ENGRG FLOW DIAGRAM CELL-D \\
\hline 71 & & 3 & 4 & INSTR ENGRG FLOW DIAGRAM CELL-D \\
\hline 72 & $\mathrm{H}-2-94504$ & 1 & 4 & INSTRUMENT ELECTRICAL FLOW DIAGRAM CELL-E \\
\hline 73 & & 2 & 2 & INSTR ENGRG FLOW DIAGRAM CELL-E \\
\hline 74 & & 3 & 4 & INSTRUMENT ELECTRICAL FLOW DIAGRAM CELL-E \\
\hline 75 & $\mathrm{H}-2-94505$ & 1 & 10 & INSTRUMENT ENGINEERING FLOW DIAGRAM CELL-F \\
\hline 76 & & 2 & 9 & INSTR ENGRG FLOW DIAGRAM CELL-F \\
\hline 77 & $\mathrm{H}-2-94506$ & 1 & 9 & INSTR ENGRG FLOW DIAGRAM CELL-G \\
\hline 78 & & 2 & 9 & INSTR ENGRG FLOW DIAGRAM CELL-G \\
\hline 79 & & 3 & 8 & INSTR ENGRG FLOW DIAGRAM CELL-G \\
\hline 80 & $\mathrm{H}-2-95984$ & 8 & 14 & ELECTRICAL ONE LINE DIAGRAM \& ELEV-MCC 16,17 \& 272B \\
\hline 81 & $\mathrm{H}-2-96494$ & 1 & 2 & FLOW DIAGRAM - AIR - BLDG 225-BC \\
\hline 82 & & 2 & 6 & FLOW DIAGRAM - AJR - BLDG 225-B \\
\hline 83 & & 3 & 0 & FLOW DIAGRAM - AIR - BLDG 225-B \\
\hline 84 & $\mathrm{H}-2-96501$ & 1 & 1 & AIR HOSE ENGRAVER \\
\hline 85 & $\mathrm{H}-2-96552$ & 1 & 4 & IEFD WESF STACK MONITOR 296-B-10 \\
\hline 86 & $\mathrm{H}-2-96557$ & 1 & 3 & WESF CAM EFD FIRST FLOOR SYSTEM C96C \\
\hline 87 & & 2 & 2 & WESF CAM EFD SECOND FLOOR SYSTEM C96C \\
\hline 88 & $\mathrm{H}-2-96639$ & 3 & 10 & ONE LINE DIAGRAM NORMAL POWER EMERGENCY POWER \\
\hline 89 & $\mathrm{H}-2-96643$ & 1 & 13 & ELECTRICAL ONE LINE DIAGRAM MCC-1 \\
\hline 90 & & 2 & 8 & ELECTRICAL ONE LINE DIAGRAM MCC-2 \\
\hline 91 & & 3 & 6 & ELECTRICAL ONE LINE DIAGRAM MCC-3 \\
\hline 92 & & 4 & 7 & ELECTRICAL ONE LINE DIAGRAM MCC-4 \& MCC-5 \\
\hline 93 & & 5 & 1 & ELECTRICAL ONE LINE DIAGRAM \\
\hline 94 & $\mathrm{H}-2.96653$ & 1 & 4 & WESF FIXED HEAD AIR SAMPLER EFD SYSTEM C96B \\
\hline 95 & $\mathrm{H}-2-99449$ & 1 & 4 & IFD EAST K3 AIR CLEANING UNIT \\
\hline 96 & & 2 & 4 & IFD WEST K3 CLEANING UNIT \\
\hline
\end{tabular}




\begin{tabular}{|c|c|c|c|c|}
\hline & Document Number & Sheet & Rev & Title \\
\hline 1 & $\mathrm{H}-2-66462$ & 1 & 6 & ELECTRICAL POWER PLAN FIRST FLOOR, AREA 1 \\
\hline 2 & $\mathrm{H}-2-66462$ & 2 & 0 & ELECTRICAL POWER PLAN FIRST FLOOR AREA 1 \\
\hline 3 & $\mathrm{H}-2-66462$ & 3 & 0 & ELECTRICAL POWER PLAN FIRST FLOOR AREA 1 \\
\hline 4 & $\mathrm{H}-2-66462$ & 4 & 0 & ELECTRICAL POWER PLAN FIRST FLOOR AREA 1 \\
\hline 5 & $\mathrm{H}-2-66462$ & 5 & 0 & ELECTRICAL POWER PLAN FIRST FLOOR AREA 1 \\
\hline 6 & $\mathrm{H}-2-66989$ & 1 & 7 & ENGINEERING FLOW DIAGRAM AQUEOUS MAKE-UP \\
\hline 7 & H-2-67081 & 1 & 8 & CELL ARRANGEMENT CELL 'A' PLAN \& ELEVATION \\
\hline 8 & $\mathrm{H}-2-67082$ & 1 & 8 & CELL ARRANGEMENT CELL 'A' ELEVATIONS \\
\hline 9 & $\mathrm{H}-2-67083$ & 1 & 10 & CELL ARRANGEMENT "B" CELL PLAN \& SECTION \\
\hline 10 & $\mathrm{H}-2-67084$ & 1 & 8 & CELL ARRANGEMENT "B" CELL SECTIONS \\
\hline 11 & $\mathrm{H}-2-67085$ & 1 & 8 & CELL ARRANGEMENT "C" CELL PLAN \\
\hline 12 & $\mathrm{H}-2-67086$ & 1 & 9 & CELL ARRANGEMENT "C" CELL SECTIONS \\
\hline 13 & $\mathrm{H}-2-67087$ & 1 & 9 & CELL ARRANGEMENT "D" \& "E" CELL PLAN \\
\hline 14 & $\mathrm{H}-2-67088$ & 1 & 9 & CELL ARRANGEMENT "D" \& "E" CELL ELEVATION \\
\hline 15 & $\mathrm{H}-2-6 \overline{7089}$ & 1 & 7 & CELL ARRANGEMENT "D" \& "E" CELL ELEVATIONS \\
\hline 16 & $\mathrm{H}-2-67090$ & 1 & 7 & CELL ARRANGEMENT F CELL PLAN \\
\hline 17 & $\mathrm{H}-2-67091$ & 1 & 9 & CELL ARRANGEMENT F CELL SECTION \\
\hline 18 & $\mathrm{H}-2-67092$ & 1 & 10 & CELL ARRANGEMENT CELL "G" PLAN \& SECTIONS \\
\hline 19 & H-2-67093 & 1 & 13 & CELL ARRANGEMENT CELL "G" ELEVATIONS \& SECTIONS \\
\hline 20 & $\mathrm{H}-2-825969$ & 2 & to & FIRE PROT CONTROLS \& BARRIERS KEY PLANS \\
\hline 21 & $\mathrm{H}-2-826287$ & 1 & 1 & INSTM INTCON DIAGRAMS \\
\hline 22 & $\mathrm{H}-2-83011$ & 1 & 1 & ELECTRICAL FACILITY ALARMS FIRST/SECOND FLOOR \\
\hline 23 & $\mathrm{H}-2-83011$ & 2 & 1 & ELECTRICAL FACILITY ALARMS FIRST FLOOR PLAN \\
\hline 24 & $\mathrm{H}-2-83011$ & 3 & 1 & ELECTRICAL FACILITY ALARMS SECOND FLOOR PLAN \\
\hline 25 & $\mathrm{H}-2-83011$ & 4 & 0 & ELECTRICAL FACILITY ALARMS SECTION VIEW \\
\hline 26 & $\mathrm{H}-2-83011$ & 5 & 10 & ELECTRICAL FACILITY ALARMS INTERCONNECT DIAGRAM \\
\hline 27 & $\mathrm{H}-2-83011$ & 6 & 0 & ELECTRICAL FACILITY ALARMS ELEMENTARY DIAGRAMS \\
\hline
\end{tabular}

